\title{
Influência da Exploração Florestal nas Populações de Espécies Ameaçadas de Extinção na FLONA do Jamari, RO
}

\author{
Evair Moreira de Souza ${ }^{1} \&$ Marta Silvana Volpato Sccoti ${ }^{1}$
}

Recebido em 09/04/2020 - Aceito em 19/07/2020

1 Universidade Federal de Rondônia, Brasil.<evairms@gmail.com, martasccoti@unir.br>.

\begin{abstract}
RESUMO - A Portaria no 443, de 17 de dezembro de 2014, reconhece 2.113 espécies da flora brasileira como ameaçadas de extinção e as classifica em quatro categorias de ameaça (extintas na natureza, criticamente em perigo, em perigo, vulnerável). As unidades de conservação desempenham importante papel para a manutenção das populações dessas espécies. Porém, essas populações podem ser afetadas mediante a influência antrópica. Nesse sentido, o presente trabalho teve por objetivo avaliar os padrões estruturais e ecológicos de espécies ameaçadas de extinção ocorrentes na Floresta Nacional do Jamari, RO, após exploração madeireira. Foram marcadas, de forma aleatória, 12 parcelas de 0,5ha em duas unidades de produção anual na unidade de manejo florestal III. Nas parcelas, inventariaram-se todos os indivíduos arbóreos com DAP $\geq 10,0 \mathrm{~cm}$. De posse da lista da composição florística obtida da amostra, foram selecionadas para o estudo as espécies constantes no livro vermelho de espécies ameaçadas de extinção e as espécies de importância econômica que ocorrem de forma restrita, de acordo com o plano de manejo da unidade de conservação. Foram gerados os descritores fitossociológicos para as espécies, taxa de mortalidade, ingresso, crescimento, padrões de distribuição espacial e estrutura diamétrica. Observaram-se na composição florística da amostra seis espécies classificadas como vulnerável (VU) e uma espécie de importância econômica com ocorrência restrita. As espécies, na sua maioria, apresentaram ocorrência rara. Não houve alterações significativas na estrutura após exploração, porém é importante monitorar e avaliar a necessidade de tratamentos silviculturais, principalmente para as espécies que constam no plano de manejo florestal.
\end{abstract}

Palavras-chave: Biodiversidade; políticas florestais; conservação.

\section{Influence of Forest Exploration on Populations of Endangered Species in FLONA do Jamari, RO}

ABSTRACT - Ordinance $n^{\circ}$ 443, of December 17, 2014 recognizes 2113 species of Brazilian flora as threatened with extinction and classifies them into four threat categories (extinct in nature, critically endangered, endangered, vulnerable). Conservation units play an important role in maintaining populations of these species. However, these populations can be affected by anthropic influence. In this sense, the present work aimed to evaluate the structural and ecological patterns of these species, post-logging, in the National Forest of Jamari, RO. Twelve plots of 0.5 ha were randomly marked in two Units of Annual Production at Forest Management Unit III, where individuals with DBH $\geq 10,0 \mathrm{~cm}$ were evaluated. With the list of the floristic composition obtained from the sample, the species listed in the book red of endangered species and species of economic importance that have low density were selected for the study, according to the conservation unit management plan. Phytosociological descriptors, mortality rate, ingress, growth, spatial distribution patterns and diametric structure. Six species classified as vulnerable (VU) and one species of economic importance with restricted occurrence. There were no changes in the structure of the species after the exploitation, however it is important to monitor and evaluate the need for the use of silvicultural treatment, especially for species included in the forest management plan.

Keywords: Biodiversity; forest policies; conservation. 


\title{
Influencia de la exploración forestal en las poblaciones de especies en peligro de extinción en FLONA do Jamari, RO
}

\begin{abstract}
RESUMEN - La Ordenanza $\mathrm{n}^{\circ}$ 443, de 17 de diciembre de 2014, reconoce 2.113 especies de la flora brasileña como en peligro de extinción y las clasifica en cuatro categorías de amenaza (extinguida en la naturaleza, en peligro crítico de extinción, vulnerable). Las unidades de conservación desempeñan un papel importante en el mantenimiento de las poblaciones de estas especies. Sin embargo, estas poblaciones pueden verse afectadas por la influencia antrópica. En este sentido, este trabajo tenía como objetivo evaluar los patrones estructurales y ecológicos de las especies en peligro de extinción que se producen en el Bosque Nacional de Jamari, RO, después de la tala. Se marcaron al azar doce parcelas de 0,5ha en dos unidades de producción anual en la unidad de ordenación forestal III. En las parcelas, todos los individuos arbóreos con DAP fueron inventariados $\geq 10.0 \mathrm{~cm}$. Con la lista de la composición florística obtenida de la muestra, se seleccionaron para el estudio las especies que figuran en el libro rojo de especies en peligro de extinción y las especies de importancia económica que se presentan de manera restringida, de acuerdo con el plan de gestión de la unidad de conservación. Se generaron descriptores fitosociológicos para la especie, la tasa de mortalidad, el ingreso, el crecimiento, las pautas de distribución espacial y la estructura diametral. En la composición florística de la muestra se observaron seis especies clasificadas como vulnerables (VU) y una especie de importancia económica de aparición restringida. La especie, en su mayoría, presentaba una ocurrencia rara. No hubo cambios significativos en la estructura después de la tala, pero es importante vigilar y evaluar la necesidad de tratamientos silvícolas, especialmente para las especies que aparecen en el plan de ordenación forestal.
\end{abstract}

Palabras clave: Biodiversidad; políticas forestales; conservación.

\section{Introdução}

Na Amazônia Legal, onde se observa a maior cobertura de florestas naturais do Brasil, ocorreu, no período de agosto de 2018 a julho de 2019, o desflorestamento de $9.762 \mathrm{~km}^{2}$ (INPE 2019). O resultado dessas ações reflete na perda de produtividade, em mudanças no regime hidrológico e na diminuição da biodiversidade (Fearnside 2005), levando muitos táxons ao processo de extinção, ou seja, ao desaparecimento de exemplares da flora ou fauna, ou de grupos dessas espécies em um determinado ecossistema.

Nesse sentido, em 2014 foi divulgada a Portaria $\mathrm{n}^{\circ} 443$, de 17 de dezembro, que reconhece as espécies da flora brasileira ameaçadas de extinção, as quais se encontram na Lista Nacional Oficial de Espécies da Flora Ameaçadas de Extinção (MMA 2014).

A metodologia utilizada para avaliação do estado de conservação das espécies brasileiras foi desenvolvida pela União Internacional para Conservação da Natureza (UICN), que utiliza dados sobre a densidade populacional, amplitude da distribuição geográfica e tamanho populacional (IUCN 2012).

Dessa forma, na Portaria $n^{\circ} 443$ são descritas quatro categorias de ameaça: as espécies extintas na natureza, as criticamente em perigo, as em perigo e as vulneráveis. Nessa lista foram apresentadas 2.113 espécies (MMA 2014), muitas delas com populações protegidas em unidades de conservação (UCs), que são áreas instituídas pelo poder público e asseguradas por legislação própria, especificada no Sistema Nacional de Unidades de Conservação (SNUC) (Brasil 2000).

As UCs se dividem em dois grupos: as unidades de proteção integral $e$ as unidades de uso sustentável. Na categoria de uso sustentável, é possível conciliar a conservação da natureza e o uso dos recursos naturais (Brasil 2000).

A Floresta Nacional do Jamari (FLONA do Jamari), em Rondônia, foi a primeira UC de uso sustentável submetida ao regime de concessão no Brasil. Na unidade, em 2008 foram licitados 96 mil hectares de área de floresta para exploração madeireira e não-madeireira (SFB 2017). Até o ano de 2018, foram transportados $253.154,64 \mathrm{~m}^{3}$ de madeira, o que gerou $\mathrm{R} \$ 14.229 .913,12$ de pagamento pela concessão (SFB 2019).

A exploração madeireira nessa área deve ser feita por meio do manejo florestal sustentável, a partir do uso de técnicas de exploração de impacto reduzido, que, quando aplicadas de forma correta, reduzem em até $50 \%$ os efeitos negativos da exploração madeireira na floresta remanescente (Putz et al. 2008). 
Porém, mesmo usando técnicas que minimizam os efeitos negativos da exploração, é importante realizar o monitoramento da floresta como forma de entender as respostas das populações de espécies ao ambiente alterado, principalmente os efeitos que isso pode causar nas populações mais frágeis.

Diante do exposto, este trabalho teve por objetivo descrever os efeitos da exploração madeireira nos padrões estruturais e ecológicos de espécies ameaçadas de extinção na Floresta Nacional do Jamari, RO.

\section{Material e Métodos}

\section{Caracterização da área de estudo}

O estudo foi desenvolvido em duas unidades de produção anual (UPAs): as UPAs 5 e a 6 , localizadas na unidade de manejo florestal III (UMF III), parte sudoeste da FLONA do Jamari, Rondônia (Figura 1).

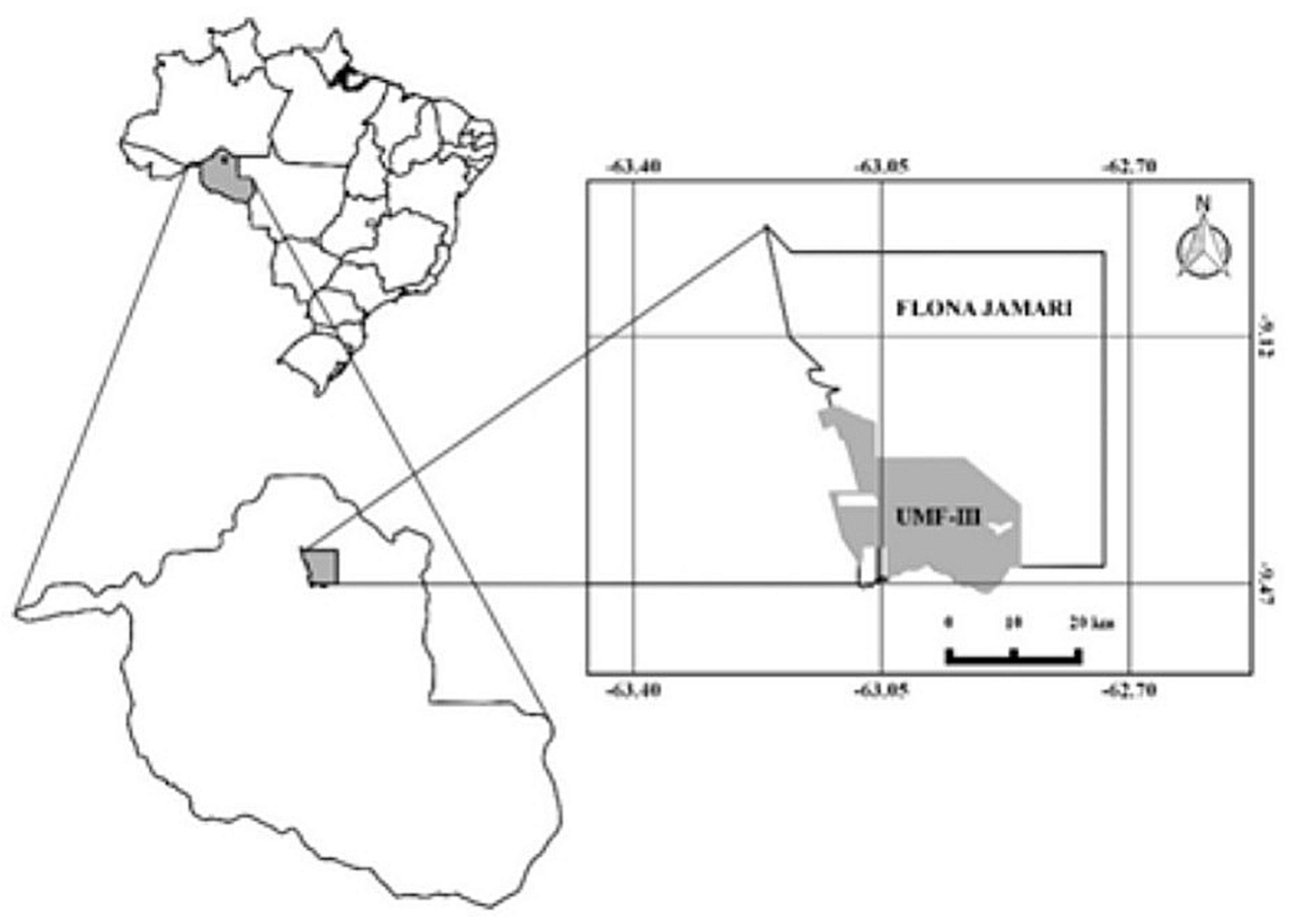

Figura 1 - Localização da unidade de manejo florestal III, FLONA do Jamari, RO. Fonte: Jacobsen (2017).

A UPA 5 possui área total de 1926,7 ha $^{-1}$ e área efetiva de exploração de $1596,69 \mathrm{ha}^{-1}$. A exploração ocorreu entre os meses de abril e novembro de 2015, a uma taxa de corte de $14,4 \mathrm{~m}^{3} \mathrm{ha}^{-1}$. AUPA 6, com área total de $1827,54 \mathrm{ha}^{-1}$ e área efetiva de exploração de $1572,44 \mathrm{ha}^{-1}$, foi explorada entre abril e outubro de 2016, com taxa de corte de $11,7 \mathrm{~m}^{3} \mathrm{ha}^{-1}$.

Segundo a classificação de Köppen, o clima da região é do tipo $\mathrm{Am}$, apresentando período de seca bem definido (Alvares et al. 2013). A temperatura média anual na região situa-se em torno de 24 e $26^{\circ} \mathrm{C}$, enquanto que a média anual de precipitação varia de 1440 a $2600 \mathrm{~mm}$.ano ${ }^{-1}$
(Rondônia 2010). O relevo da área é formado, na sua maioria $(87,7 \%)$, por altitude inferior a $150 \mathrm{~m}$ (Amata 2007).

A tipologia florestal predominante é a Floresta Ombrófila Aberta (ISA 2018), e as espécies com maior valor de importância observadas na área são Protium robustum (Swart) D.M.Porter, Pseudolmedia laevis (Ruiz \& Pav.) J.F.Macbr., Eschweilera pseudodecolorans S.A.Mori, Tachigali Aubl., Pouteria torta (Mart.) Radlk., Beilschmiedia brasiliensis (Kosterm.) Kosterm., Copaifera multijuga Hayne, Peltogyne paniculata Benth., Apeiba echinata Gaertn., Astronium lecointei Ducke, Bertholletia excelsa 
Bonpl., Dinizia excelsa Ducke, entre outras (Sccoti 2016, Jacobsen 2017).

\section{Coleta de dados}

Antes da exploração madeireira, em cada UPA foram marcadas, de forma aleatória, parcelas permanentes de $100 \mathrm{~m} \times 50 \mathrm{~m}$, onde se avaliaram todos os indivíduos arbóreos com diâmetro à altura do peito $(\mathrm{DAP}) \geq 10 \mathrm{~cm}$. Na UPA 5 foram marcadas sete parcelas, e os inventários ocorreram em março de 2015 e outubro de 2016. Na UPA 6 foram avaliadas cinco parcelas em março de 2016 e outubro de 2017. Assim, para este estudo foram utilizados dados de 12 parcelas permanentes, perfazendo uma área amostral de 6ha.

A marcação e medição das parcelas permanentes seguiu a metodologia proposta por Silva et al. (2005), que determinam as diretrizes técnicas para a marcação e medição de parcelas permanentes no bioma Amazônia.

Após doze meses do término das atividades de exploração, realizou-se um segundo inventário nas parcelas permanentes, a fim de caracterizar a composição e a estrutura da vegetação pósexploração.

Foram coletados dados de DAP, altura comercial e total, qualidade do fuste, iluminação da copa e qualidade da copa.

\section{Seleção das espécies ameaçadas de extinção}

De posse dos dados do inventário florístico/ fitossociológico das parcelas permanentes, selecionaram-se as espécies que constam no Livro Vermelho da Flora no Brasil (Martinelli \& Moraes 2013), Portaria $n^{\circ} 443$, de 17 de dezembro de 2014, e as espécies de importância econômica que ocorrem de forma restrita, segundo o plano de manejo da unidade de conservação (MMA/IBAMA 2005).

\section{Análises dos dados}

A análise das mudanças causadas após exploração na população das espécies selecionadas foi feita por meio da estrutura fitossociológica, definida pelo cálculo dos estimadores clássicos da fitossociologia (densidade absoluta, dominância absoluta, frequência absoluta), taxas de ingresso, mortalidade $e$ incremento periódico em DAP para o período de 19 meses (Souza \& Soares 2013).

Para verificar diferenças estatísticas na estrutura populacional das espécies após exploração, utilizou-se o teste $t$ de Student para amostras pareadas a $5 \%$ de probabilidade. Para o conjunto de dados que não apresentaram normalidade pelo teste de Kolmogorov-Smirnov, foi aplicado o teste não paramétrico de MannWhitney a $5 \%$ de probabilidade. As variáveis utilizadas para aplicação dos testes estatísticos foram abundância e dominância. Os testes ocorreram no programa estatístico Bio Stat 5.0 (Ayres et al. 2007).

Para caracterizar os padrões de distribuição espacial das espécies na área, foi utilizado o Índice de Agregação de Morisita (IM) (Barros et al. 1984). O padrão espacial é considerado agregado quando o IM>1. Usou-se o teste de Qui-quadrado para avaliar diferenças estatísticas quanto ao padrão aleatório de dispersão (Barros et al. 1984).

Por fim, gerou-se a caracterização da estrutura diamétrica das espécies por meio do número de indivíduos amostrados por classe de DAP. Utilizaram-se intervalos de $10 \mathrm{~cm}$ para as classes.

\section{Resultados}

$\mathrm{Na}$ área de estudo, foram encontradas seis espécies listadas no Livro Vermelho da Flora no Brasil, sendo elas: castanheira (Bertholletia excelsa Bonpl.), cedro (Cedrela fissilis Vell.), garapeira (Apuleia leiocarpa (Vogel) J.F.Macbr.), Itaúba (Mezilaurus itauba (Meisn.) Taub. ex Mez), Jatobá (Hymenaea parvifolia Huber) e breu-gigante (Protium giganteum Engl.), todas classificadas como vulnerável (Tabela 1). E uma espécie, macaúba (Platymiscium duckei Huber), constante na lista de espécies de grande importância econômica que apresentam ocorrência restrita, conforme plano de manejo da unidade de conservação (MMA/IBAMA 2005).

Dentre as espécies observadas, Bertholletia excelsa foi a que apresentou o maior número de indivíduos por hectare (1,33ind.ha-1 ${ }^{-1}$, distribuídos em $50 \%$ das parcelas avaliadas (Tabela 2). Protium giganteum teve redução na densidade populacional após exploração, causada pela morte de indivíduos; porém, manteve-se como 
Tabela 1 - Espécies florestais ameaçadas de extinção observadas em parcelas permanentes em unidade de manejo florestal na Floresta Nacional do Jamari, RO, Brasil.

\begin{tabular}{|c|c|c|c|}
\hline Família & Nome científico & Nome comum & Categoria \\
\hline Caesalpiniaceae & Apuleia leiocarpa (Vogel) J.F.Macbr.* & Garapeira & VU \\
\hline Lecythidaceae & Bertholletia excelsa Bonpl.* & Castanheira & VU \\
\hline Meliaceae & Cedrela fissilis Vell.* & Cedro & VU \\
\hline Lauraceae & Mezilaurus itauba (Meisn.) Taub. ex Mez ${ }^{*}$ & Itaúba & VU \\
\hline Fabaceae & Hymenaea parvifolia Huber & Jatobá & VU \\
\cline { 2 - 4 } & Platymiscium duckei Huber ** & Macaúba & RARA \\
\hline Burseraceae & Protium giganteum Engl. ${ }^{*}$ & Breu-gigante & VU \\
\hline
\end{tabular}

* Espécies listadas no Livro Vermelho de Espécies Ameaçadas de Extinção no Brasil, Portaria n ${ }^{\circ} 443$, de 17 de dezembro; ** espécie de importância econômica que ocorre de forma restrita, conforme plano de manejo da unidade de conservação (MMA/IBAMA 2005).

a segunda espécie com maior densidade. Já Cedrela fissilis apresentou menor densidade antes da exploração e após as atividades de exploração, seguida por Platymiscium duckei.
Essas alterações não indicaram diferenças significativas $(\mathrm{P}>0,05)$ na estrutura populacional após exploração (Tabela 2).

Tabela 2 - Parâmetros fitossociológicos antes e após exploração de espécies ameaçadas de extinção observadas em parcelas permanentes em unidade de manejo florestal na Floresta Nacional do Jamari, RO, Brasil.

\begin{tabular}{|c|c|c|c|c|c|c|c|c|c|c|c|c|}
\hline \multirow{2}{*}{ Espécie } & \multicolumn{5}{|c|}{ Antes da exploração } & \multicolumn{5}{|c|}{ Após a exploração } & \multicolumn{2}{|c|}{ p-valor } \\
\hline & $\mathbf{N}$ & pi & DA & FA & DoA & $\mathbf{N}$ & pi & DA & FA & DoA & Abun. & DoA \\
\hline Apuleia leiocarpa & 2 & 1 & 0.33 & 8.3 & 0.38 & 2 & 1 & 0.33 & 8.3 & 0.38 & 1 & 0.339 \\
\hline Bertholletia excelsa & 8 & 6 & 1.33 & 50 & 0.70 & 8 & 6 & 1.33 & 50 & 0.73 & 1 & 0.177 \\
\hline Cedrela fissilis & 1 & 1 & 0.17 & 8.3 & 0.05 & 1 & 1 & 0.17 & 8.3 & 0.05 & 1 & 0.339 \\
\hline Hymenaea parvifolia & 2 & 2 & 0.33 & 16.7 & 0.06 & 2 & 2 & 0.33 & 16.7 & 0.06 & 1 & 0.249 \\
\hline Mezilaurus itauba & 3 & 3 & 0.5 & 25 & 0.03 & 3 & 3 & 0.5 & 25 & 0.03 & 1 & $0.977 *$ \\
\hline Platymiscium duckei & 2 & 2 & 0.33 & 16.7 & 0.03 & 1 & 1 & 0.17 & 8.3 & 0.01 & 0.339 & 0.339 \\
\hline Protium giganteum & 7 & 4 & 1.17 & 33 & 0.02 & 4 & 4 & 0.67 & 33 & 0.01 & $0.8174^{*}$ & $0.863^{*}$ \\
\hline
\end{tabular}

Onde: $\mathrm{N}$ = número de indivíduos observados na amostra; $\mathrm{pi}=$ número de parcelas em que se observou a ocorrência da espécie; $\mathrm{DA}=$ densidade absoluta (ind.ha $\left.{ }^{-1}\right) ; \mathrm{FA}=$ frequência absoluta (\%); DoA= dominância absoluta $\left(\mathrm{m}^{2} \mathrm{ha}^{-1}\right) ;$ Abun. = abundância. * Teste de Mann-Whitney a $5 \%$ de probabilidade.

A espécie Bertholletia excelsa ainda evidenciou maior dominância absoluta entre as espécies estudadas, ocupando $0,70 \mathrm{~m}^{2} \mathrm{ha}^{-1}$ antes da exploração e $0,73 \mathrm{~m}^{2} \mathrm{ha}^{-1}$ após a exploração. Esse valor de dominância absoluta foi influenciado pela maior densidade de plantas e maior valor médio de diâmetro observado para a espécie $(68,45 \mathrm{~cm} \pm 50,61 \mathrm{~cm})$.
Duas espécies apresentaram mortalidade no período de 19 meses: Platymiscium duckei e Protium giganteum (Tabela 3). Ressalta-se que as causas da mortalidade foram decorrentes das atividades de exploração para ambas as espécies. Nenhuma espécie apresentou indivíduos ingressantes, e o maior crescimento médio em diâmetro foi registrado para Bertholletia excelsa (1,6cm em 19 meses). 
Tabela 3 - Taxa de Mortalidade (TM), ingresso (TI), incremente periódico (IP) e padrão de distribuição espacial de espécies arbóreas ameaçadas de extinção observadas em parcelas permanentes em unidade de manejo florestal, na FLONA do Jamari, RO, Brasil.

\begin{tabular}{|l|c|c|c|c|c|}
\hline \multicolumn{1}{|c|}{ Espécies } & TM(\%) & TI(\%) & IP DAP(cm) & IM & $\chi^{2} \mathbf{c a l}$ \\
\hline Apuleia leiocarpa & 0 & 0 & 0,7 & 4,00 & 20,0 \\
\hline Bertholletia excelsa & 0 & 0 & 1,6 & 2,0 & 14,0 \\
\hline Cedrela fissilis & 0 & 0 & 0,1 & 0 & 8,0 \\
\hline Hymenaea parvifolia & 0 & 0 & 0,75 & 0 & 8,0 \\
\hline Mezilaurus itauba & 0 & 0 & 0,63 & 0 & 8,0 \\
\hline Platymiscium duckei & 50,00 & 0 & 0,0 & 0 & 8,0 \\
\hline Protium giganteum & 42,86 & 0 & 0,44 & 4,57 & 21,7 \\
\hline
\end{tabular}

Onde: $\mathrm{TM}=$ taxa de mortalidade; $\mathrm{TI}=$ taxa de ingresso; $\mathrm{IP}=$ incremente periódico em diâmetro a altura do peito $(\mathrm{DAP}) ; \mathrm{IM}=$ índice de Morisita; $\chi^{2}$ cal $=$ teste de Qui-quadrado calculado; $\chi^{2} \operatorname{tab}(11 ; 0,05)=19,675$.

Quanto ao padrão de distribuição espacial das espécies avaliadas, quatro apresentaram padrão uniforme (IM $=0)$, sendo elas: Cedrela fissilis, Hymenaea parvifolia, Mezilaurus itauba e Platymiscium duckei; no entanto, ressalta-se que essas espécies tiveram baixo número de indivíduos amostrados nas parcelas. Cedrela fissilis, por exemplo, foi representada por apenas um indivíduo; Hymenaea parvifolia e Platymiscium duckei, por 2 indivíduos cada; e Mezilaurus itauba por 3 , sendo que cada indivíduo ocorreu em parcelas diferentes, denotando caráter mais uniforme.
As espécies Apuleia leiocarpa e Protium giganteum apresentaram padrão de distribuição agrupado, confirmado pelo valor de qui-quadrado calculado $\left(\chi^{2} \mathrm{cal} \geq \chi^{2} \mathrm{tab}\right)$ com nível de 0,05 de probabilidade; já a espécie Bertholletia excelsa apresentou padrão aleatório ( $\mathrm{IM}=2,0 ; \chi^{2} \mathrm{cal}<\chi^{2} \mathrm{tab}$ no nível 0,05 de probabilidade).

As espécies mostraram distribuição diamétrica descontínua, com exceção de Bertholletia excelsa, que apresentou estrutura mais estável; além disso, a estrutura se manteve a mesma após a exploração (Figura 2).

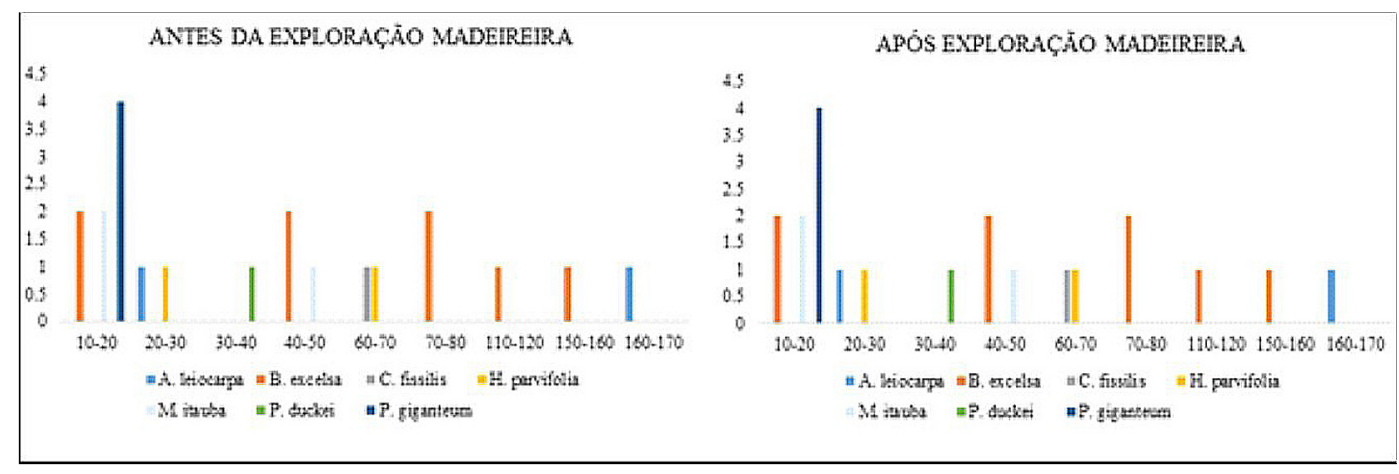

Figura 2 - Número de indivíduos por classe de diâmetro a altura do peito (DAP), antes e após a exploração madeireira, para espécies arbóreas ameaçadas de extinção observadas em parcelas permanentes em unidade de manejo florestal, na FLONA do Jamari, RO, Brasil.

\section{Discussão}

No presente estudo, seis espécies foram descritas como protegidas (Apuleia leiocarpa,
Bertholletia excelsa, Cedrela fissilis, Hymenaea parvifolia, Mezilaurus itauba, Protium giganteum e Platymiscium duckei) pela Portaria $\mathrm{n}^{\circ} 443$, de 17 de dezembro de 2014, Livro Vermelho da Flora 
no Brasil, e pelo plano de manejo da unidade de conservação (MMA/IBAMA 2005).

Essas espécies também foram descritas em outros estudos no bioma Amazônico, com valores de densidade, frequência e dominância próximos aos verificados neste trabalho (Condé \& Tonini 2013, Andrade et al. 2017), denotando a importância da região Amazônica para a manutenção e conservação desses grupos de espécies ameaçadas.

As atividades de manejo florestal afetaram a estrutura de duas espécies, Protium giganteum e Platymiscium duckei, que tiveram a densidade absoluta reduzida devido à mortalidade causada por danos decorrentes da exploração madeireira.

Gomes (2018) observou o comportamento de espécies pertencentes à categoria vulnerável após exploração florestal e aplicação de tratos culturais na Floresta Nacional do Tapajós. Uma das espécies estudadas foi Hymenaea parvifolia, a qual apresentou redução na abundância, frequência e dominância após um ano da exploração em função da colheita de dois indivíduos e, seis anos após a exploração, a espécie já havia recuperado a abundância e a frequência, mas não a dominância, que só apresentou recuperação do estoque na avaliação feita 30 anos após a exploração.

A autora ainda concluiu que o fato de a espécie constar na lista das espécies ameaçadas de extinção não significa que deva sofrer restrições ao corte, pois, aplicando-se técnicas adequadas, ela pode ser explorada e se manter conservada na área em manejo.

Diante disso, por serem espécies em categoria de vulnerabilidade, é importante prever nos planos de manejo florestal o monitoramento desses grupos e estratégias de exploração com menor impacto e tratos culturais que possam promover a regeneração natural e, principalmente, o crescimento das árvores remanescentes.

O corte de cipós é uma técnica recomendada para promover o crescimento e a qualidade tecnológica da madeira (Tafarel et al. 2014, Souza et al. 2015), podendo ser prevista na etapa préexploratória no manejo florestal, com o objetivo de evitar acidentes e danos ao produto durante o abate das árvores.

De forma geral, estudos em áreas que foram submetidas à exploração de baixo impacto apontaram baixa influência das atividades de exploração na estrutura geral das populações de espécies na floresta e recomposição do estoque explorado (Souza et al. 2017, Castro et al. 2018). Isso demonstra a importância do uso de técnicas adequadas para a exploração florestal, principalmente em unidades de conservação, que são locais que abrigam não só as espécies ameaçadas de extinção, mas como grande parte da biodiversidade local.

A legislação não obriga o uso de técnicas de impacto reduzido nos planos de manejo na Amazônia; no entanto, estudos têm apontado que os danos na floresta são significativamente menores quando comparados às técnicas convencionais de exploração, assim como a recuperação da floresta é mais rápida (Sogobal et al. 2000, Silva 2004, Bulfe et al. 2008).

Dentre espécies estudadas, Bertholletia excelsa foi a de maior relevância em termos estruturais, pois apresentou maior densidade, dominância, crescimento e estrutura diamétrica mais estável. Os valores observados foram próximos aos encontrados em trabalho sobre estrutura populacional dessa espécie no Acre (Neves et al. 2016). Os autores ainda citam que o manejo sustentável de produtos florestais não-madeireiros é preconizado pela existência de uma estrutura populacional estável para a espécie de interesse.

Segundo Souza \& Soares (2013), a estabilidade das populações na floresta está associada à quantidade de indivíduos que a espécie apresenta em todas as classes de tamanho, possibilitando o recrutamento contínuo.

Bertholletia excelsa, além da importância ecológica que tem para o ecossistema florestal, é relevante para a econômica local, por ser uma das principais espécies fornecedoras de produtos florestais não madeireiros na região Norte (Balzon et al. 2014, Pedrozo et al. 2011, Silva et al. 2013), atuando como um elemento da floresta responsável pela manutenção do extrativismo realizado por comunidades da região. Assim, é fundamental que sua estrutura esteja em condições de estabilidade, como apontam os resultados deste trabalho.

Em áreas de concessão florestal, a espécie é excluída do objeto de uso e não pode ser explorada pelo concessionário por se tratar de produto de uso tradicional de subsistência das comunidades locais, podendo as empresas apenas promover a exploração pelas comunidades do entorno 
da unidade de conservação (Brasil 2006), o que evidencia a importância da manutenção das populações dessa espécie para a região Amazônica, assim como a necessidade da sua conservação na floresta.

Na área de estudo, Bertholletia excelsa apresentou padrão aleatório de distribuição espacial, padrão também descrito por Tonini et al. (2008) no sul do estado de Roraima, e por Coelho et al. (2015) em estudo no sudeste do Pará.

Segundo Tonini et al. (2008), as sementes de castanheira são dispersas principalmente pela cutia, podendo ser consumidas imediatamente pelo seu agente dispersor, como armazenadas para posterior consumo ou abandonadas em outras áreas onde acabam germinando. Peres \& Baider (1997) complementam que as sementes de castanheira podem ser dispersas a até $25 \mathrm{~m}$ de distância da árvore matriz e são enterradas individualmente a uma profundidade de 1 a $3 \mathrm{~cm}$.

Outra espécie de grande valor de mercado observada no levantamento foi Apuleia leiocarpa, que apresentou baixa densidade e estrutura diamétrica descontínua, assim como Hymenaea parvifolia e Mezilaurus itauba. As espécies possuem alto potencial econômico, sendo os principais alvos da exploração ilegal na região da FLONA (https:// www.bvrio.org/leilao-madeira), e podendo, a longo prazo, desaparecer da composição florística dos tipos florestais da Amazônia se não forem tomadas medidas preventivas de controle ao desmatamento $e$ à exploração ilegal.

De forma geral, percebe-se que as espécies com baixa densidade representam importante função no ecossistema, seja na geração de biodiversidade, como na atuação expressiva na economia local. Leitão et al. (2016) ressaltam que as espécies com baixa ocorrência são as primeiras a se tornarem extintas após distúrbios induzidos pelo homem, e que a diversidade de características funcionais dentro de grupos de espécies conduz a vários processos ecológicos relevantes para a manutenção dos ecossistemas.

\section{Conclusão}

$\mathrm{Na}$ FLONA do Jamari, seis espécies protegidas como vulneráveis (Apuleia leiocarpa, Bertholletia excelsa, Cedrela fissilis, Hymenaea parvifolia, Mezilaurus itauba, Protium giganteum) foram observadas em área de exploração madeireira, e uma foi classificada como de importância econômica de ocorrência restrita (Platymiscium duckei), conforme plano de manejo da unidade de conservação.

A exploração florestal realizada na área não afetou significativamente a estrutura populacional das espécies estudadas; no entanto, o monitoramento das populações se faz importante para avaliar os estoques a longo prazo $e$ a necessidade de tratos silviculturais, principalmente daquelas espécies que foram exploradas.

As unidades de conservação representam habitat que ainda conservam essas espécies classificadas como ameaçadas de extinção, e a realização de pesquisas, principalmente em áreas destinadas ao uso, são fundamentais para orientar gestores dessas unidades, assim como a elaboração de políticas públicas de conservação da biodiversidade.

\section{Agradecimentos}

Ao Instituto Chico Mendes de Conservação da Biodiversidade (ICMBio)/Unidade Itapuã do Oeste, por conceder a área de estudo; e à Empresa Amata, pelo apoio logístico para a realização do trabalho.

\section{Referências}

AMATA. 2007. Plano de Manejo Florestal Sustentável. 142p.

Ayres M, Ayres Júnior M, Ayres DL \& Santos AAS. 2007. Bioestat 5.0: Aplicações estatísticas nas áreas das ciências biológicas e médicas. Belém: MCT; IDSM; CNPQ. 380p.

Alvares CA, Stape JL, Sentelhas PC, de Moraes G, Leonardo J \& Sparovek G. Köppen's climate classification map for Brazil. Meteorologische Zeitschrift, 22(6): 711-728, 2013.

Andrade RT, Pansini S, Sampaio AF, Ribeiro MS, Cabral GS \& Manzatto AG. Fitossociologia de uma floresta de terra firme na amazônia Sul-Ocidental, Rondônia, Brasil. Biota Amazônia, 7: 36-43, 2017.

Balzon DR, Silva JC \& Santos AJ. Aspectos mercadológicos de produtos florestais não madeireiros Análise retrospectiva. Floresta, 34(3): 363-371, 2014.

Barros PLC \& Machado SA. 1984. Aplicação do índice de dispersão em espécies de florestas tropicais da Amazônia Brasileira. FUPEF. 44p. 
Brasil. 2000. Lei $\mathrm{n}^{\circ}$ 9.985. Dispõe sobre sistema nacional de unidades de conservação (SNUC). http:// www.planalto.gov.br/ccivil_03/leis/L9985.htm. Acesso em: 31/05/2018.

Brasil. 2006. Lei $\mathrm{n}^{\circ}$ 11.284. A gestão de florestas públicas para a produção sustentável. http://www.mma. gov.br/port/conama/legiabre.cfm?codlegi $=485$. Acesso em: 08/05/ 2018.

Bulfe NML, Galvão F, Figueiredo Filho A \& Donagh PM. Efeitos da exploração convencional e de impacto reduzido em uma floresta estacional semidecidual na província de Misiones, nordeste da Argentina. Floresta, 39(2): 365-379, 2009.

Castro MAS, Viana APdaS, Lobato CC, Muniz, JL, Santos MWdos \& Melo, LdeO. Impactos da exploração florestal na estrutura e na composição florística da vegetação remanescente em área manejada na Floresta Nacional do Tapajós, Belterra, Pará. Agroecossistemas, 10(2): 125-135, 2018.

Coelho FD, Pinto MP, Neto PN, Gomes JM, Soares MH \& Ruschel AR. Estrutura populacional da castanheira (Bertholletia excelsa) no parque zoobotânico de Marabá, Pa. Anais do XIII Seminário Anual de Iniciação Científica da UFRA. 2015. https://ainfo.cnptia.embrapa. br/digital/bitstream/item/137098/1/Estrutura1.pdf. Acesso em: 15/01/2018.

Condé TM \& Tonini H. Fitossociologia de uma floresta ombrófila densa na amazônia setentrional, Roraima, Brasil. Acta Amazonica, 43(3): 250-260, 2013.

Fearnside PM. Deforestation in Brazilian Amazonia: History, Rates and Consequences. Conservation Biology, 19(3): 680-688, 2005.

Gomes JM. 2018. Dinâmica das populações de três espécies madeireiras ameaçadas de extinção na Amazônia Oriental. Tese (Doutorado em Ciências Florestais). Universidade Federal Rural da Amazônia. 89p.

INPE (Instituto Nacional de Pesquisas Espaciais). 2019. A estimativa da taxa de desmatamento por corte raso. $<$ http://www.inpe.br/noticias/noticia.php?Cod Noticia $=5294>$. Acesso em: 08/04/2020.

ISA. Instituto Socioambiental. Flona do Jamari. 2018. $<$ https://uc.socioambiental.org/pt-br/uc/3332>. Acesso em: 05/06/2018.

IUCN (International Union for Conservation of Nature's). IUCN red list categories e criteria: The IUCN Red List of Threatened Species, 2012. <www.iucnredlist.org/ technical-documents/categories-and-criteria $>$. Acesso em: 22/03/2018.

Jacobsen RHF. 2017 Impactos na vegetação após corte seletivo em área de concessão florestal na Amazônia Ocidental. Dissertação (Mestrado em Ciências
Ambientais). Fundação Universidade Federal de Rondônia. 70p.

Leitão RP et al. Rare species contribute disproportionately to the functional structure of species assemblages. The Royal Society Publishing, 283: 1-6, 2016. < https://royalsocietypublishing.org/doi/pdf/10.1098/ rspb.2016.0084>. Acesso em: 10/06/ 2018.

Lima $\mathrm{B}$ de $\mathrm{A}$ et al. Estrutura e dinâmica florestal sob efeito do manejo madeireiro na Flona do Tapajós. Advances in Forestry Science, 5(4): 437-443, 2018.

Martinelli G \& Moraes MA. 2013. Livro vermelho da flora no Brasil. CNFLORA. Rio de Janeiro, RJ, Brasil. 1100p.

Matteucci SD \& Colma A. 1982. Metodologia para el estudio de la vegetacion. Washington: Secretaria General de la Organizacion de los Estados Americanos - Programa Regional de Desarrollo y Tecnologico. $169 \mathrm{p}$.

MMA/IBAMA. (Ministério do Meio Ambiente/Instituto Brasileiro de Meio Ambiente e dos Recursos Naturais Renováveis). 2005. Plano de Manejo da Floresta Nacional do Jamari. 135p. <https://www.icmbio.gov. br/portal/images/stories/docs-planos-de-manejo/flona_ jamari_pm_anexos.pdf>. Acesso em: 08/2018.

MMA (Ministério do Meio Ambiente). 2014. Portaria $n^{\circ} 443$. Atualização da lista de espécies ameaçadas de extinção da flora brasileira. <http://cncflora.jbrj.gov. br/portal/static/pdf/portaria_mma_443_2014.pdf $>$. Acesso em: 08/04/2020.

Neves EdeS, Wadt LHdeO \& Guedes MC. Estrutura populacional e potencial para o manejo de Bertholletia excelsa (Bonpl.) em castanhais nativos do Acre e Amapá. Scientia Forestalis, 44(109): 19-31, 2016.

Pedrozo EÁ, Silva TN, Sato SA \& Oliveira ND. Produtos florestais não madeiráveis (PFNMS): As filières do açaí e da castanha da Amazônia. Revista de Administração e Negócios da Amazônia, 3: 88-112, 2011.

Peres CA \& Baider C. Seed dispersal, spatial distribuition and population structure of Brazil nut trees (Bertholletia Excelsa) in Soltheastern Amazônia. Journal of Tropical Ecology, 13(4): 595-616, 1997.

Putz FE et al. Improved tropical forest management form carbon retention. Plos Biology, 6(7): 1368-1369, 2008.

Rondônia. 2010. Secretaria de Estado do Desenvolvimento Ambiental (SEDAM). Boletim Climatológico de Rondônia, ano 2008. SEDAM, Porto Velho. 36p.

Sccoti MSV. 2016. Análise da dinâmica da vegetação após exploração na concessão florestal da Flona do Jamari, RO. Relatório de Pesquisa Projeto CNPQ. 39p. 
SFB. Serviço Florestal Brasileiro. Balanço de produção, Jamari, RO, 2017. http://www.florestal.gov.br/florestassob-concessao/96-concessoes-florestais/florestas-sobconcessao/jamari/370-balanco-de-producao-jamari. Acesso em: 20/05/2018.

SFB. Serviço Florestal Brasileiro. Balanço de produção, Jamari, RO, 2019. http://www.florestal.gov.br/florestassob-concessao/96-concessoes-florestais/florestas-sobconcessao/jamari/370-balanco-de-producao-jamari. Acesso em: 08/04/2020.

Silva EJV. Dinâmica de florestas manejadas e sob exploração convencional na Amazônia oriental. 2004. Tese (Doutorado em Ciências da Engenharia Ambiental). Universidade de São Paulo. 156p.

Silva AA, Santos MK, Gama RJ, Vasconcelos R \& Leão S. Potencial do extrativismo da Castanhado-Pará na geração de renda em comunidades da mesorregião baixo Amazonas, Pará. Floresta e Ambiente, 20(4): 500-509, 2013.

Silva JNM et al. 2005. Diretrizes para instalação e medição de parcelas permanentes em florestas naturais da Amazônia Brasileira. Belém, PA: Embrapa Amazônia Oriental. 68p.

Souza AL \& Soares CB. 2013. Floresta Nativa: estrutura, dinâmica e manejo. Editora: UFV. 322p.
Sabogal C, Silva JNM, Zweede J, Pereira Júnior R, Barreto P \& Guerreiro CA. 2000. Diretrizes técnicas para a exploração de impacto reduzido em operações florestais de terra firme na Amazônia brasileira. Embrapa Amazônia Oriental. 52p.

Souza DV, Carvalho JOPde, Mendes FdaS, Melo LdeO, Silva JNM \& Jardim FCdaS. Crescimento de espécies arbóreas em uma floresta natural de terra firme após a colheita de madeira e tratamentos silviculturais, no município de Paragominas, Pará, Brasil. Ciência Florestal, 25(4): 873-883, 2015.

Souza MS, Azevedo CP, Souza CR, França M \& Neto ELV. Dinâmica e produção de uma floresta sob regime de manejo sustentável na Amazônia central. Floresta, 47(1): 55-64, 2017.

Taffarel M, Carvalho JOPde, Silva LdeO, Silva MGda, Gomes JM \& Ferreira JER. Efeito da silvicultura póscolheita na população de Lecythis lurida (miers) mori em uma floresta de terra firme na Amazônia brasileira. Ciência Florestal, 24(4): 889-898, 2014.

Tonini H, Costa P \& Kaminski PE. Estrutura e produção de duas populações nativas de castanheira-do-brasil em Roraima. Floresta, 38(3): 445-457, 2008.

\section{Biodiversidade Brasileira - BioBrasil.}

Edição de Fluxo Contínuo

n. 3,2020

http://www.icmbio.gov.br/revistaeletronica/index.php/BioBR

Biodiversidade Brasileira é uma publicação eletrônica científica do Instituto Chico Mendes de

Conservação da Biodiversidade (ICMBio) que tem como objetivo fomentar a discussão $e$ a disseminação de experiências em conservação e manejo, com foco em unidades de conservação $e$ espécies ameaçadas. 